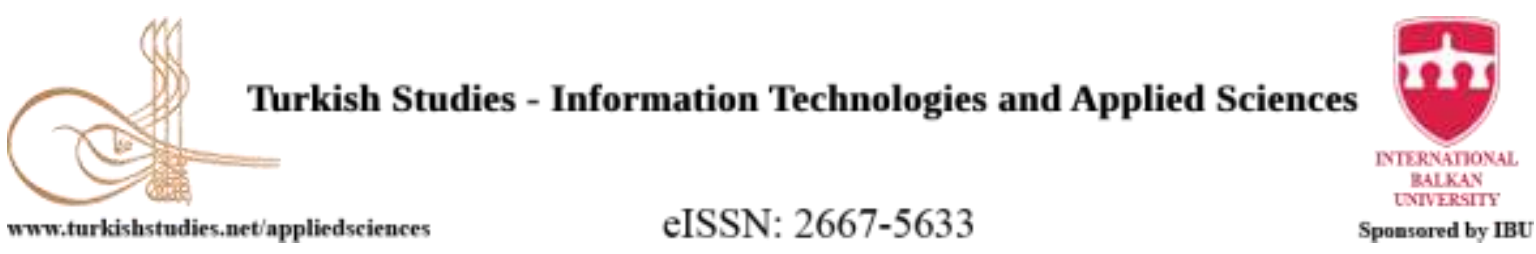

Research Article / Araştırma Makalesi

\title{
MRI Kardiyak MR Görüntülerinde Sol Kulakçık Bölütlenmesi İçin Derin Öğrenme Tabanlı Hibrit Model
}

\author{
Deep Learning Based Hybrid Model for Left Atrial Segmentation in Cardiac
}

\author{
İlkay Öksüz*
}

\begin{abstract}
Accurate left atrial (LA) segmentation from cardiac magnetic resonance imaging (MRI) is one of the key challenges for achieving higher precision atrial fibrillation ablation, quantification of fibrosis and enabling electromechanical modelling of the heart. However, due to motion blur caused by heart movement and inconsistent intensity patterns near the atrial border, automating left atrial segmentation is a challenging task. In this work, we propose a hybrid approach, where we combine a convolutional neural network with conditional random fields to fully automatically delineate the LA from cardiac volumetric MRI. We first generate a coarse segmentation using U-net architecture and then apply conditional random fields to effectively integrate contextual and appearance information on LA cardiac volumes. Our method is capable to generate a smooth 3D atrial geometry with the regularization of conditional random fields. We trained and tested the proposed method on 100 3D cardiac MRI datasets from the STACOM 2018 atrial segmentation challenge and showed that it outperforms single U-net and its variants both in 2D and 3D. Dice coefficient, Jaccard Similarity Index and Hausdorf metric were used as validation metrics to evaulate the segmentation. For testing cases the Dice metric, Jaccard Similarity Index and Hausdorf metric were computed as $0.912 \pm 0.0680 .927 \pm 0.097$ and $18.54 \pm 4.21$ respectively.
\end{abstract}

Structured Abstract: Due to its superiority in soft tissue contrast and no risk of exposure to radiation, Magnetic Resonance Imaging (MRI) is becoming the most commonly used imaging modality in the world. Cardiac magnetic resonance (CMR) imaging is essential in determining the cardiac health and cardiac tissue viability for radiologists. Accurate delineation of the chambers is an important task and automatic algorithms are taking important roles within the clinical practice. One key challenge for CMR images is automatic segmentation of left atrium to aid atrial fibrillation ablation and atrial tissue characterization. The algorithms that are developed for this task must overcome certain challenges such as indefinite atrial borders, low image contrast and motion blurring. To this end, we propose an algorithm that combines deep learning methods with conditional random fields to accurately delineate left atrium. Our algorithm is tested on MICCAI 2018 atrial segmentation challenge and the improvements achieved with conditional random fields are illustrated both quantitatively and qualitatively.

Recent success of neural network-based algorithms in computer vision tasks motivated the medical image analysis community to utilize similar approaches for disease classification and anatomic segmentation. Deep learning methods and fully convolutional neural networks have been successfully applied to segmentation

\footnotetext{
* Dr. Öğr. Üyesi, Istanbul Teknik Üniversites, Bilgisayar-Bilişim Fakültesi, Bilgisayar Mühendisliği Bölümü, Asst. Prof. Dr., Istanbul Technical University, Computer Engineering Department ORCID 0000-0001-6478-0534

oksuzilkay@itu.edu.tr

Cite as/ Atıf: Öksüz, İ. (2020). MRI Kardiyak MR görüntülerinde sol kulakçık bölütlenmesi için derin öğrenme tabanlı hibrit model. Turkish Studies - Applied Sciences, 15(3), 361-371. https://dx.doi.org/10.47844/TurkishStudies.45551

Received/Geliş: 01 Augost/Ağustos 2020

Accepted/Kabul: 20 September/Eylül 2020

Checked by plagiarism software

Copyright $\odot$ INTAC LTD, Turkey

Published/Yayın: 25 September/Eylül2020

CC BY-NC 4.0
} 
of anatomical structures in various medical image modalities. For CMR most of the recently proposed precise techniques rely on fully convolutional neural networks. Encoder-Decoder type architectures are capable of learning features in multiple scales in an efficient way and especially skip-connections have been instrumental for reinforcing the information from early layers in the final output. U-net architecture has set the state of the art for an increasing number of medical image segmentation problems and has proven to be effective in many grand challenges in top venues. However, when inconsistencies in boundaries exist the final output of the 2dimensional algorithm needs refinement to match the clinically acceptable delineations. Moreover, some anatomical structures do not exhibit clear intensity boundaries, which challenges the automatic algorithms in producing smooth anatomical contours.

In this paper, we propose to use a fully convolutional neural network architecture (U-net) in combination with conditional random fields to achieve smooth segmentation with high accuracy. Our hybrid method is capable of addressing the shortcomings of inconsistent boundaries by regularizing the effect of random fields. We have done a variety of ablation studies to showcase the performance difference in between 2D and 3D U-net architecture. Furthermore, we have utilized both 2D and 3D CRF refinement on the output of U-net to illustrate the superior performance of incorporating information from third dimension. We have tested our algorithm on cardiac volumetric MRI datasets from the STACOM 2018 atrial segmentation challenge and showed that it outperforms single U-net and its variants. The training dataset of the challenge consists of 100 cardiac MRI scans. For, our training is done using 80 percent of the data and testing is done on the remaining 20 percent. The validation data is randomly selected as 20 percent of all training to data to adjust the parameters. Our training is done using Dice metric as the loss function. We have utilized 10 times data augmentation to enrich the dataset and avoid over-fitting. Our loss function is Dice loss, which aims to increase the overlap in between the algorithm output and ground truth segmentation. We utilize Adam optimizer with a learning rate of $10^{-3}$ and reduce the learning rate if the Dice loss does not improve for 10 consecutive epochs. For evaluation, we have utilized three different metrics namely: Dice metric, Jaccard index and Hausdorf distance. In all three metrics we have compared the 2D and 3D approaches. The 3D hybrid method showcased superior performance compared to $2 \mathrm{D}$ and 3D U-net architectures. Moreover, we compared the 2D and 3D applications of the hybrid model and illustrated the superiority of the 3-dimensional approach. We also showcase qualitatively the smoothness of the final output of our algorithm compared to the 2D U-net approach.

Our study shows that combining information from neural networks with random fields can improve the segmentation of left atrium volumetric cardiac MRI data. Moreover, incorporating 3D information can aid achieving smooth and consistent contours of left atrium in cardiac MRI. This technique can be utilized in segmentation different anatomical structures of various image modalities. One avenue of improvement for our algorithm is including disease classification to our framework. In the availability of records on the patient's conditions coupled with imaging data, our algorithm can be adjusted to output disease classification. The clinical translation of such techniques can aid radiologists in daily practice both in terms of accuracy and time in disease diagnosis.

Keywords: Computer Science and Engineering, Machine Learning, Atrial Segmentation, Cardiac MR, Deep Learning, Conditional Random Fields

Öz: Kardiyak manyetik rezonans görüntülerinde (MRG) başarılı sol kulakçık (SK) bölütlemesi daha yüksek hassasiyetli atriyal fibrilasyon ablasyonu, SA fibroz ölçümü ve kardiyak elektromekanik modelleme elde etmek için önemli zorluklardan biridir. Bununla birlikte, kardiyak MR dan otomatik SK bölütlemesi kalbin dinamik hareketi ve sınır noktalarındaki yeğinlik değeri farkları, görüntü çözünürlüğü, denekler arasındaki anatomik yapılarda önemli değişkenlik nedeniyle zordur. Bu çalışmada, SK'nın kardiyak volumetrik MRG'den tam otomatik bölütlenmesi için 3-boyutlu birleşik derin öğrenme ve rastgele alanlar yaklaşımı önerilmiştir. İlk önce U-net mimarisini kullanarak kaba bir SK bölütlmesi elde edilmiştir. Daha sonra son SK bölütlemesi için kardiyak hacimlerdeki bağlamsal ve görünüm bilgilerini etkili bir şekilde entegre etmek için koşullu rastgele alanlar uygulanmıştır. Yöntem rastgele alanlar sayesinde 3-boyutlu düzenlenmiş ve pürüzsüz bir sol karıncık yüzeyi sonucu vermektedir. STACOM 2018 veritabanında 100 adet kardiyak volumetrik MRG veri kümesi üzerinde önerilen yöntemi doğrulanmıştır. Önerilen yöntem sadece U-net yöntemini uygulamaktan daha iyi performans göstermiştir. Ayrıca 2-boyutlu ve 3-boyutlu yöntem karşılaştırılması yapılarak, 3-boyutlu yaklaşımın SK yüzeyi için sağladığı iyi performans nicel ve nitel olarak gösterilmiştir. Doğrulama ölçütü olarak, ortalama Dice katsayısı, Jaccard Benzerlik Katsayısı skoru ve Hausdorff Mesafesi kullanılmıştır.

Turkish Studies - Applied Sciences, 15(3) 
Önerlien yöntemin test verileri üzerinde Dice Katsayısı $0.912 \pm 0.068$, Jaccard Benzerlik Katsayısı skoru

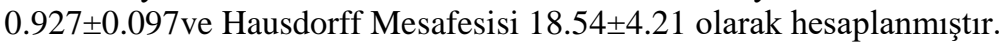

Anahtar Kelimeler: Bilgisayar Bilimleri ve Mühendisliği, Makine Öğrenmesi, Kulakçık Bölütlemesi, Kardiyak MR, Derin Öğrenme, Rastgele Alanlar

\section{Giriş}

Düzensiz ve hızlı bir kalp hızına yol açan atriyal fibrilasyon (AF), en sık görülen kalp ritmi bozukluğudur. AF 60 yaşın üzerindeki kişilerde 5 inmenin l'ine neden olur ve önemli ölüm nedenlerinden biridir (Narayan vd., 2017). AF için mevcut klinik tedaviler, insan atriyumunda AF'yi doğrudan ilgilendiren atriyal anatomik yapının temel anlayışının eksikliği nedeniyle kötü performans göstermektedir (Zhao vd., 2017). Kronik AF rahatsızlığı her iki karıncığa geniş bir şekilde dağılmış kompleks alt tabakalar tarafından yönlendirilir (Hansen vd., 2017). Tekrarlanan AF atakları ayrıca atriyumun yapısal özelliklerinde, yani atriyal yapısal yeniden şekillenmede çeşitli değişiklik üretir (Narayan \& Zaman., 2016). Sonuç olarak, atriyal yapının ve AF hastalarındaki değişikliklerin doğrudan incelenmesi AF'nin anlaşılması ve tedavisi için hayati öneme sahiptir.

Günümüzde gadolinyum tabanlı kontrast maddeleri, hastanın Manyetik Rezonans (MR) taramalarının üçte birinde, kalp dahil olmak üzere hastanın iç yapılarının görüntülerinin netliğini artırmak için kullanılmaktadır. Geç gadolinyumla güçlendirilmiş manyetik rezonans görüntüleme (LGE-MRI), kardiyak fibroz / skar oluşumu ve dağılımını incelemek için yaygın olarak kullanılmaktadır (Zhao vd., 2017a, 2017b). LGE-MRI kullanan AF hastalarında yapılan klinik çalışmalar, atriyal fibrozisin kapsamının ve dağılımının kateter ablasyon başarı oranının güvenilir birer öngörücüsü olduğunu ve tıbbi tedavi için hasta sınıflandırmasında kullanılabileceğini düşündürmektedir (Higuschi vd., 2017). Geniş bir klinik çalışma olan LGE-MRI Kılavuzlu Ablasyon ve Atriyal Fibrilasyonun Konvansiyonel Kateter Ablasyonu kalpteki fibroz bölgesini gösteren LGEMRI tarafindan yönlendirilen kateter ablasyon tedavisine karşı konvansiyonel kateter ablasyon tedavisini değerlendirmektedir. Ayrıca, 3D LGE-MRG'lerden hesaplanan sol kulakçık (SK) çap ve hacim klinik tanı ve tedavi tabakalandırması için güvenilir bilgi sağlar (Njoku vd., 2018).

Kulakçık bölütlemesi, segmentli 3B geometrinin yapısal analizine dayanarak AF hastaları için tıbbi tedaviye yardımcı olmak için çok önemli bir işlemdir. SK için tam otomatik atriyal bölütleme algoritmaları araştıran önceki çalışmalar mevcuttur. Tobon-Gomez ve arkadaşları (TobonGomez vd., 2013) gadolinyum içermeyen MR / BT'den SK bölütlemesi için dokuz farklı algoritmanın performansını karşılaştırmış ve istatistiksel modelleri bölgesel büyüme yaklaşımlarıyla birleştiren metodolojilerin en etkili olduğunu göstermiştir. Veni ve arkadaşlarının çalışmalarında, LGE-MRI'lardan SA'nın segmentlere ayrılması için benzer teknikler de önerilmiş ve geliştirilmiştir (Veni vd., 2017, Tao vd., 2016). Mevcut yapısal analiz çalışmalarının çoğu, özellikle klinik LGEMRG'leri kullananlar, hala emek-yoğun ve hataya eğilimli elle işaretleme yaklaşımlarına dayanmaktadır (Njoku vd., 2018). Bunun başlıca nedeni, başlatma için şekil öncelikleri (Veni vd., 2013) veya bölütleme sürecine yardımcı olmak için ek manyetik rezonans anjiyografi (MRA) sekansları gibi sıklıkla mevcut olmayan destekleyici bilgileri gerektiren mevcut otomatik metodolojilerin sınırlamalarından kaynaklanmaktadır. Kıyaslama çalışmasında arkadaşları (TobonGomez vd., 2013) belirtilen dokuz algoritma SK'1 gadolinyum içermeyen MR'lardan ayırmada etkili olmuştur. Bununla birlikte, (normal) atriyal anatomi kontrast madde tarafindan daha fazla zayıflatıldığı ve geleneksel yaklaşımlara dayandığı için LGE-MRI'lara doğrudan uygulanması da zordur. Bu nedenle, klinik kullanım için atriyal geometrinin doğru rekonstrüksiyonu ve ölçümü için doğrudan LGE-MRI'lardan, özellikle SK için, tam otomatik atriyal bölütleme gerçekleştirebilen akı1lı bir algoritmaya ihtiyaç ortaya çıkmıştır.

Sınıflandırma ve bölütleme, makine öğrenmesi algoritmalarıyla çözülen iki tipik sorundur. Destek vektör makineleri (Suykenes vd., 1999) ve rastgele ormanlar (Ho vd., 1995) gibi geleneksel 
makine öğrenimi sınıflandırma algoritmalarında, ham verilerden manuel olarak bir dizi özellik oluşturulur ve bir sınıflandırıcıya beslenir. Bu, öğrenme için en iyi özellik kombinasyonunu bulmak için titiz bir özellik seçim prosedürü gerektiğinden, eldeki görev alanında etki alanı uzmanlığı gerektirir. Daha sonra objektif bir işlevi en aza indirmek için özellikler üzerinde optimizasyon yapılır, bu da verilerin farklı sinıflardan doğrusal olarak ayrılmasına neden olur. Bununla birlikte, bu algoritmaların yıllar içindeki etkinliğine rağmen, manuel özellik mühendisliği ve algoritma seçim süreçleri, sınıflandırma görevlerindeki performansı iyileştirmek için önemli darboğazlardır. Sinir ağları (LeCun vd., 2015) modern makine-öğrenme algoritmalarının bir kategorisidir ve tıp (Papik vd., 1998, Shahid vd., 2019), ve biyomühendislik (Park vd., 2018) dahil birçok farklı alanda uygulanmıştır. Sinir ağlarının etkinliği özellik çıkarma adımını otomatikleştirme yeteneklerinde yatmaktadır. Sinir ağları uygulanırken alan uzmanlığı ihtiyacını ortadan kaldırarak, bu algoritmaların performansı ancak mevcut veri miktarı arttıkça artacaktır. On yıldan kısa bir süre önce popüler hale gelen evrişimsel sinir ağları (ESA) (LeCun vd., 1998), görüntü sınıflandırması (Szegedy vd., 2015, He vd., 2016), nesne algılama (Ren vd., 2015) ve bölütleme (Noh vd., 2015) gibi görüntü işleme konusunda uzmanlaşmıştır. Böylece, ESA kulakçık bölütlemesi zorlu görevini çözmek için ideal bir temel sağlar. Derin öğrenme yöntemleri SK bölütleme üzerinde çeşitli temellerde uygulanmaya başlanmış ve umut verici sonuçlar elde edilmiştir (Xiong vd., 2020). Bu makalede evrişimsel sinir ağlarını rastgele alanlar ile birlikte kullanabilen ve tamamen otomatik SK bölütlemesi yapabilen bir algoritma öneriyor ve değerlendiriyoruz. Önerilen yöntemde STACOM 2018 yarışması (Xiong vd., 2020) dahilindeki 100 3D LGE-MRI veri seti üzerinde geliştirilmiş ve doğrulanmıştır. Bu heyecan verici gelişme hastaya özel teşhis ve tedaviye doğru çok önemli bir adımdır.

\section{Metotlar}

Bu bölüm kullandığımız veri ve metotlar hakkındaki detaylardan bahsetmektedir. İlk önce MICCAI 2018 çerçevesinde düzenlenen yarışmada kullanılan sol kulakçık versini tanıtılmıştır. Daha sonra verilerde yaptığımız ön işlemeden bahsedeceğiz ve en son olarak kullandığımız yapay sinir ağı modelini ve rastgele alanlar yöntemlerini tanıtılmıştır.

\section{Veriseti}

Bu makalede, algoritmamız 2018 Atriyal Segmentasyon Yarışmasının veri seti (Xiong vd., 2020) üzerinde eğitildi ve değerlendirildi. Bu veri seti, 100 adet 3B gadolinyum ile geliştirilmiş manyetik rezonans görüntüleme taramasından oluşmaktadır. Ayrıca bu görüntülere karşılık gelen uzmanlar tarafından çizilmiş SK manuel bölütleme maskeleri mevcuttur. Veri setinin \%80'ni eğitim \%20'si ise test için ayrılmıştır. Ayrıca eğitim setinin yüzde 20'si parametre değerlendirmesi için kullanmıştır. Bu şekilde çapraz gerçekleme kullanarak veride bölütleme sonuçları aldık. Ağımızın eğitimi veya ön eğitimi için herhangi bir harici veri kullanmadık.

\section{Önişleme}

Bu veri kümesindeki görüntüler yarışma organizasyonu tarafından yeniden örneklendi ve ön işleme tabi tutuldu. Dolayısıyla, ön işleme aşamasında yeniden örnekleme prosedürüne gerek yoktur. Verilerin çözünürlüğünde gözlemlenen tutarlılığa rağmen, bu veri kümesi görüntü boyutları ve görüntü kontrastında büyük farklılıklar gösterir. Örneğin, MRG veri setimizde iki boyut resmi vardır: eksenel düzlemlerde $576 \times 576$ ve $640 \times 640$. Bunun dışında, atria, farklı görüntülerde, çeşitli şekil ve boyutlara da sahip olabilir. Bu fenomenler, bu taramaların farklı tarayıcılara ve görüntüleme protokollerine sahip olabilen birden fazla yerden toplanması nedeniyle ortaya çıkabilir. Bu nedenle, bu görüntüler için sağlam bir yöntem oluşturmak önemlidir. Şekil 1, farklı görünümlerde farklı görüntülerin görüntü kontrastındaki farkı gösterir. $\mathrm{Bu}$ makalede, ele alınmış olan farklı görüntülerdeki genelleme yeteneğini geliştirmek amacıyla veri çeşitliliğini artırmak için veri arttırımını kullanıyoruz. 


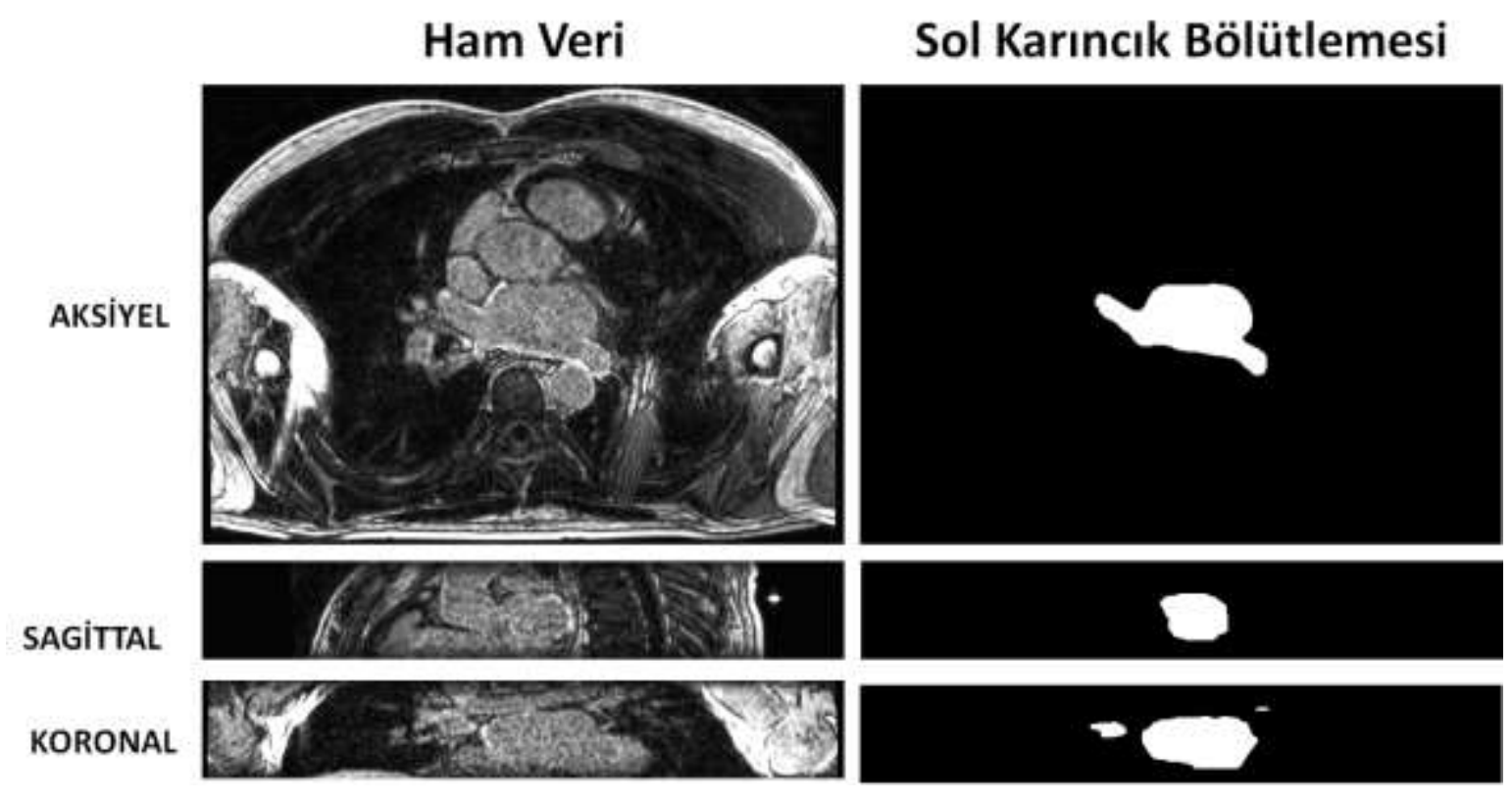

Şekil 1: Örnek Kardiyak MR verisi ve uzman tarafından işaretlenmiş sol kulakçık bölütlemesi. Üç ayrı düzlemde 3-Boyutlu görüntün 2-Boyutu kesitleri gösterilmiştir.

Görüntülerin çözünürlügünü korumak için veri ön işleme aşamasında görüntü yeniden ölçeklendirmesi yapılmamıştır. Bunun yerine, veri çeşitliliğini artırmak için çok ölçekli kırpma kullanıldı, böylece ağ görüntüleri farklı şekilde analiz edebilir. Uygulanan önemli ön işleme tekniği bütün vücut görüntülerinden kalbe ilişkin bölgenin çıkarılmasıdır. Bu işlem için şablon eşleştirme yönteminden faydalanılmıştır:

$$
\frac{\sum_{x, y}\left[f(x, y)-f_{u, v}^{\prime}\right]\left[t(x-u, y-v)-t^{\prime}\right.}{\sqrt{\sum_{x, y}\left[f(x, y)-f_{u, v}^{\prime}\right] \sum_{x, y}[t(x-u, y-v)]}}
$$

$\mathrm{Bu}$ denklemde $\mathrm{f}$ görüntüyü, $\mathrm{t}$ şablonu temsil etmektedir. $\mathrm{t}^{\varsigma}$ şablonun ortalamasını, $f_{u, v}^{\prime}$ de aranan küçük temsili alanın ortalama yeğinlik değerini temsil etmektedir.. Hem eğitim hem de test aşamasında ön işleme aşamasındaki gerekli adım, görüntü yeğinliğini, ortak uygulamada yaygın olarak kabul edilen sıfır ortalamaya ve birim varyansa normalleştirmektir.

\section{Derin Öğrenme}

Bu bölümde, önerilen ağımızın mimarisini ve nihai 3B bölütleme maskesini almak için ağ çıktısını nasıl işlediğimizi sunuyoruz. Önerilen ağımız, ağın derinliğini artırdığımız ve bir sınıflandırma dalı eklediğimiz bir U-Net mimarisinden (Ronneberger vd., 2015) etkilenmiştir. Biyomedikal görüntü segmentasyonu için evrişimsel bir ağ olan U-Net mimarisinin detayları Şekil 2'de verilen formatı, kardiyak MR görüntülerin segmentasyonu için kullanılmıştır. A ğ bir kodlayıc1 (encoder) ve genişleyen bir kod-çözücü (decoder) yollardan oluşur. Kodlayıcı yolunda, her biri rektifiye edilmiș doğrusal birim (ReLU) aktivasyon fonksiyonu kullanmaktadır. Her bir katmanda 3 tane 3X3X3 filtreleri olan evrişimsel sinir ağları vardır. Her katmanın sonunda 2x2X2 havuzlama yöntemi uygulanmıştır, bu sayede her adımda, kanalı sayısı yarıya düşer Mimarinin fazla 
yakınsamasını önlemek için kodlayıcı kısmında her adımında yüzde 20 oranında seyreltme (dropout) kullanılmıştır. Son katmanda çıktıyı sınıf sayısına eşlemek için bir 1X1 filtre kullanan evrişimsel sinir ağı kullanılmıştır.

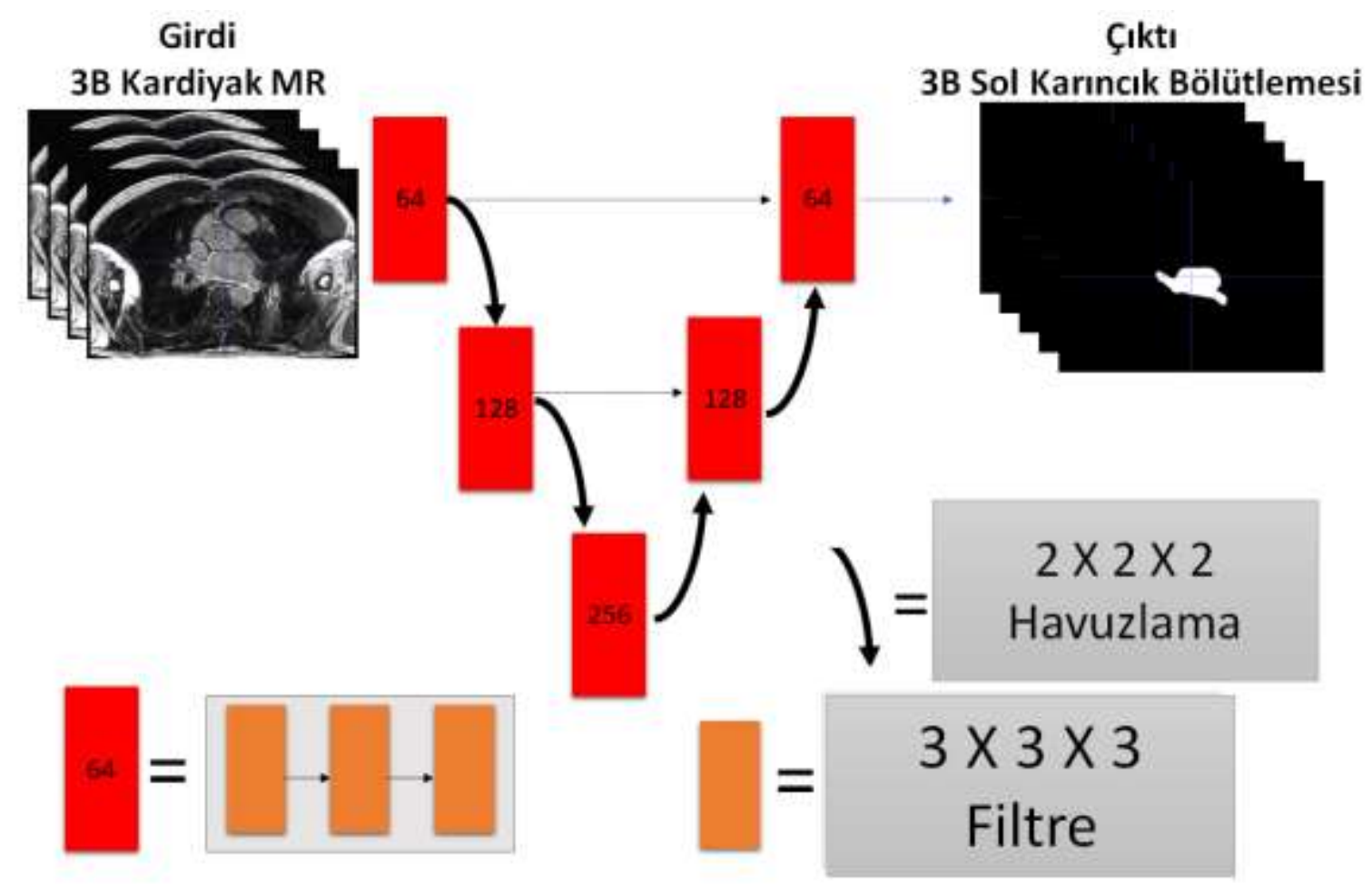

Şekil 2: Kardiyak sol kulakçık bölütlemesi için U-net Model yapısı. Kırmızı kutular evrişimsel sinir ağı bloklarını temsil etmektedir. Kırmızı kutuların içindeki rakamlar filtre sayılarını temsil etmektedir. Kesik çizgiler alt katmanlardan gelen direk bağlantıları göstermektedir.

NVIDIA Quadro RTX 6000 GPU üzerindeki Pytorch kütüphanesini kullanarak U-Net mimarisini eğittik. Eğitim, 5 küme boyutuyla yaklaşık 2 saat sürer. Eğitim için Dice hata fonksiyonu kullanılmıştır. Hem eğitim setindeki hem de test setindeki her görüntünün piksel değerlerini sıfır ortalaması olan piksel değerlerine normalleştirdik. Öğrenme oranı $10^{-3}$ olarak ayarlandı. Optimizasyon algoritması olarak ADAM algoritması (Kingma \& Ba, 2014) kullanılmıştır. Öğrenme oranının çok küçük seçilmesi uzun bir eğitim dönemine neden olabilirken, büyük bir değer çok hızlı veya dengesiz bir eğitim sürecinin öğrenilmesine yol açabilir. Bu problemden kaçınmak için, her 10 döngüde dice skoru iyileştirme göstermezse öğrenme oranı 0,5 ile çarpılarak azaltılmıştır.

\section{Veri Arttırma}

Eğitim verilerimiz, görüntü dönüşümlerinin bir bileşimi ile artırıldı, \% 50 olasılıkla rastgele yatay / dikey çevirme, -10 ila +10 derece aralığında rastgele döndürme, orijinal görüntü boyutunun yüzde 10'u aralığında $\mathrm{X}$ ve $\mathrm{Y}$ ekseni boyunca rastgele kaydırma ve arasında bir faktörle yakınlaştırma dahil 0.7 ve 1.3 kullanılmıştır. Eğitim verisini 10 kat arttıracak şekilde veri eklmesi yapılmıştır. Veri kümesinde, düşük kontrastlı efektlerin bir görüntünün görsel kalitesini düşürebilecek ve böylece bölütleme doğruluğunu etkileyebilecek çeşitli görüntü kontrastları mevcuttur. Bu sorunu çözmek için, geleneksel makine öğrenme yöntemleri genellikle görüntü ön işleme sırasında görüntü kontrast1 geliştirme yöntemleri kullanır. Burada, eğitim sırasında farklı kontrast seviyelerine sahip çeşitli 
görüntüler üretmek için gama düzeltmesine dayalı bir kontrast büyütme yöntemi önerdik. Bu şekilde ESA'mız, görüntü kontrastı farkından bağımsız olarak görüntüleri bölümlere ayırma becerisi kazanabilir ve test sirasında herhangi bir kontrast ayarlamas1 yapmaya gerek yoktur. Bu nedenle, yöntemimiz bu ayarlamaları gerektiren genel geleneksel yöntemlerden daha verimlidir. Çeşitli veri arttırma yöntemi örnekleri Şekil 3'de verilmiştir.

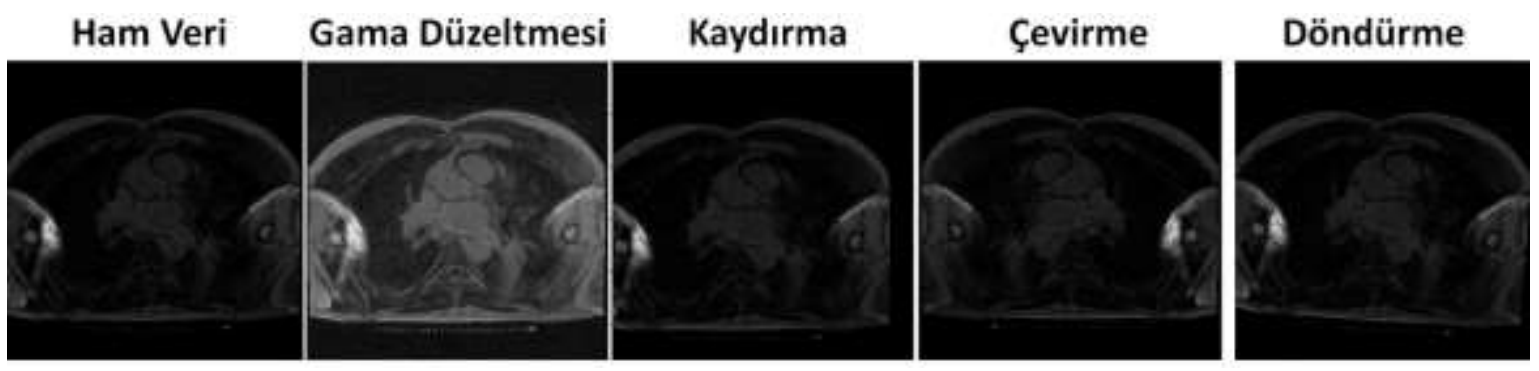

Şekil 3: Çeşitli veri arttırma örnekleri

\section{Son İşleme}

U-net mimarisinden aldığımız çıktıların başarımını arttırmak için rastgele alanlar uygulanarak bölütleme kalitesi arttırılmıştır. U-Net'ten çıkan yumuşak tahminler (olasılık haritaları) verildiğinde nihai etiket atamasını yoğun bir Şartlı Rastgele Alan'da maksimum posteriori (MAP) çıkarım olarak formüle ederek hem lokasyon tutarlılığını hem de görünümü dikkate almamızı sağliyoruz.

Tüm görüntüyü bir grafik olarak tanımlıyoruz $G=(V, E)$. Ardından yoğun $C R F$ 'yi belirtiyoruz resimdeki her voksel $i \in V$ ve kenarlar için köşeleri ile $e_{i j} \in E=\{(i, j) \forall i, j \in V, i<j$ için $\}$ olarak tanımlıyoruz.. Değişken vektörü $\mathrm{x} \in \mathrm{L}^{\mathrm{N}}$, her noktadaki i $\in \mathrm{V}$ etiketi tanımlamaktadır (Örneğin 1 sol karıncık, 0 dğer). Gibbs dağılımına göre uyarlanan enerji fonksiyonu daha sonra şu şekilde verilir:

$$
E(x)=\sum_{i \in \mathrm{V}} \phi_{i}\left(x_{i j}\right)+\sum_{(i, j) \in \mathrm{E}} \phi_{i j}\left(x_{i}, x_{j}\right)
$$

En iyi etiketlemeyi tahmin etmek için $\mathrm{x}^{*}=\arg \min _{\mathrm{x} \in \mathrm{LN}} \mathrm{E}(\mathrm{x})$ verimli kullanarak ortalama alan yaklaşım algoritması uygulanmıştır. CRF'nin ağırlıkları ve çekirdekleri rastgele bir arama algoritması kullanılarak seçildi (Krahebühl vd., 2011).

\section{Deneysel Bulgular}

$\mathrm{Bu}$ bölümde yaptığımız deneyleri ve ulaştırılan sol kulakçık bölütleme başarılarını raporluyoruz. İlk kısımda kullanılan başarı metrikleri tanıtılmıştır. Daha sonra, karşılaştırma için kullanılan yöntemler tanıtılmış ve nicel sonuçlar raporlanmıştır. En son olarak da çeşitli şekillerle sonuçlar görselleştirilmiştir.

Tablo 1: Bölütleme Sonuçları. Veri arttırma (VA) ve rastgele alanlar (RA) daha başarılı sonuçlar elde edilmesini sağlamıştır.

\begin{tabular}{cccc}
\hline Yöntem & Dice & JBK & HM (piksel) \\
2B U-Net & $0.819 \pm 0.109$ & $0.794 \pm 0.091$ & $27.17 \pm 7.21$ \\
3B U-Net & $0.825 \pm 0.134$ & $0.841 \pm 0.097$ & $24.88 \pm 7.04$ \\
\hline 2B U-Net+VA & $0.833 \pm 0.116$ & $0.818 \pm 0.121$ & $25.17 \pm 6.19$ \\
3B U-Net+VA & $0.848 \pm 0.129$ & $0.855 \pm 0.104$ & $23.08 \pm 6.61$ \\
\hline 2B U-net+2B RA & $0.883 \pm 0.085$ & $0.890 \pm 0.131$ & $21.08 \pm 5.84$ \\
3B U-net+3B RA & $\mathbf{0 . 9 1 2 \pm 0 . 0 6 8}$ & $\mathbf{0 . 9 2 7 \pm 0 . 0 9 7}$ & $\mathbf{1 8 . 5 4 \pm 4 . 2 1}$ \\
\hline
\end{tabular}


Farklı deneysel ayarlar için bölütleme doğruluğunu değerlendirmek için üç ölçüm kullanıyoruz: Dice skoru (Dice benzerlik katsayısı skoru olarak da bilinir), Jaccard Benzerlik Katsayısı (JBK) skoru ve Hausdorff Mesafesi (HM). Sonuçlar Tablo 1'de gösterilmektedir. 10 kat veri arttırma 2B ve 3B U-net modelinin performanslarını arttırmıştır. 3B U-net algoritması her 3 metrik için de 2D U-nete göre daha iyi sonuçlar vermektedir. Bunun temel sebebi sol karıncığın anatomik yapısının 3 boyutta daha iyi anlamlandırılmasıdır. Ayrıca rastgele alanlar hem 2B hem de 3B uygulamalarında son işleme olarak kullanıldğı durumlarda daha iyi sonuç vermiştir. Özelliklerde yüzeylerde yarattığı avantaj HM karşılaştırmasında gözlemlenmektedir.

Özellikle Mitral kapak düzlemlerinde ağın derinliği arttırılarak bölütleme performansının büyük ölçüde iyileştirildiği görülebilir (Şekil 5). En iyi sonuçlarımız, Derin U-Net ve ardından rastgele orman yöntemi kullanılarak 0.912'lik bir Dice skoru elde edildi. Şekil 4'teki görselleştirme grafiklerinden, önerilen modelin U-Net algoritmasından daha başarılı olduğunu görebiliyoruz. Şekil 3, modelimizin 3B segmentasyon sonucumuz ve uzman bölütlemesi karşılaştırmalı olarak verilmiştir. Şekil 5'de ise 3B rastgele alanların bölütleme yüzeyi üzerine etkisi gösterilmiştir. 2B analizlerde oluşan girinti ve çıkıntı yapıları 3B algoritmalarda gözlenmektedir.
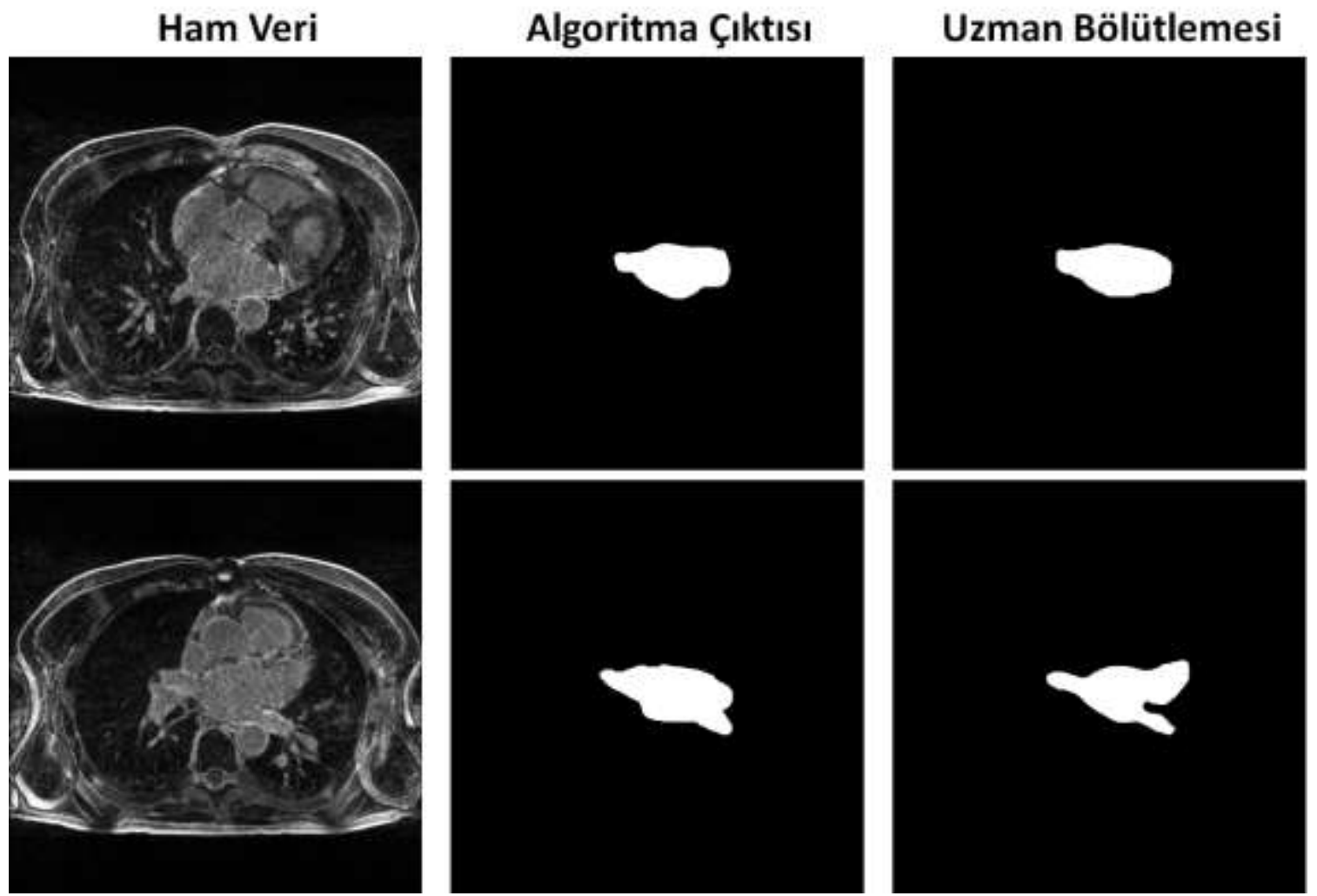

Şekil 4: Örnek Kardiyak MR bölütleme sonucu ve uzman bölütlemesi 


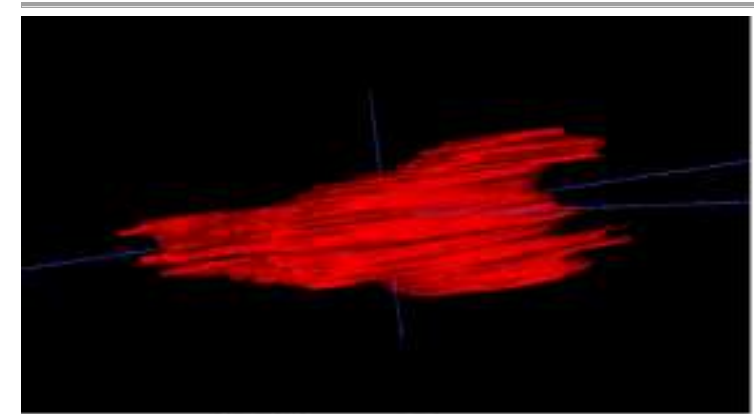

2B U-net Sonucu

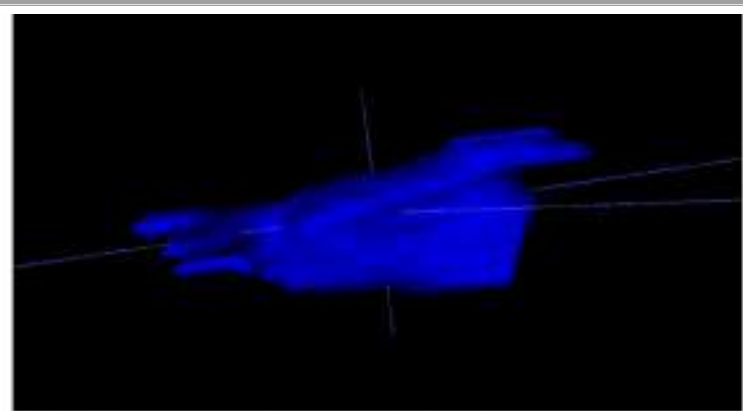

3B U-net+ Rastgele Alanlar Sonucu

Şekil 5: Örnek Kardiyak MR 2B U-net sonucu ve 3B U-net+Rastgele Alanlar Sonucu. Rastgele alanlar daha düzgün bir yüzey oluşumunu sağlamıştır.

\section{Sonuç}

Bu makalede, sol karıncı ğı otomatik olarak GE-MRG'lerinden ayırmak için derin bir 3B tam evrişimsel sinir ağı önerdik. ESA ve RA yöntemleri hibrit şekilde kullanarak, ağımız temel U-Net yöntemine kıyasla gelişmiş bölütleme doğruluğu gösterdi. Ek olarak, rastgele alanların, çeşitli görüntü kontrastlı görüntüleri analiz ederken modelimizin başarısını ve verimliliğini artırmanın etkili ve etkili bir yolu olduğunu gösterdik. Ayrıca çeşitli veri arttırma yöntemlerinin ESA modelinin SK bölütlemesi performansını arttırdığı deneylerle doğrulanmıştır. Modelimizi ileriki çalışmalarda daha fazla veri üzerinde eğitip çeşitli kliniklerden alınan veriler üstünde doğrulama yapma amaçlanmaktadır. Bu klinik sorunlar için temel bir problem olan genelleştirme problemine cevap verme noktasında başarılı bir yöntem oluşturulmasını sağlayacaktır.

\section{Teşekkür}

$\mathrm{Bu}$ araştırma, Türkiye Bilimsel ve Teknolojik Araştırma Kurumu (TÜBİTAK) tarafından 118C353 numaralı proje kapsamında desteklenmiştir.

\section{Kaynakça}

Hansen, B. J., Zhao, J., \& Fedorov, V. V. (2017). Fibrosis and atrial fibrillation: computerized and optical mapping: a view into the human atria at submillimeter resolution. JACC: Clinical Electrophysiology,3(6), 531-546.

He, K., Zhang, X., Ren, S., \& Sun, J. (2016). Deep residual learning for image recognition. In Proceedings of the IEEE conference on computer vision and pattern recognition (pp. 770778).

Higuchi, K., Cates, J., Gardner, G., Morris, A., Burgon, N. S., Akoum, N., \& Marrouche, N. F. (2018). The spatial distribution of late gadolinium enhancement of left atrial magnetic resonance imaging in patients with atrial fibrillation. JACC: Clinical Electrophysiology, 4(1), 49-58.

Ho, T. K. (1995, August). Random decision forests. In Proceedings of 3rd international conference on document analysis and recognition (Vol. 1, pp. 278-282). IEEE.

Kingma, D. P., \& Ba, J. (2014). Adam: A method for stochastic optimization. arXiv preprint arXiv:1412.6980.

Krähenbühl, P., \& Koltun, V. (2011). Efficient inference in fully connected crfs with gaussian edge potentials. In Advances in neural information processing systems (pp. 109-117).

LeCun, Y., Bottou, L., Bengio, Y., \& Haffner, P. (1998). Gradient-based learning applied to document recognition. Proceedings of the IEEE, 86(11), 2278-2324. 
LeCun, Y., Bengio, Y., \& Hinton, G. (2015). Deep learning. Nature, 521(7553), 436-444.

Narayan, S. M., Rodrigo, M., Kowalewski, C. A., Shenasa, F., Meckler, G. L., Vishwanathan, M. N., ... \& Wang, P. J. (2017). Ablation of focal impulses and rotational sources: What can be learned from differing procedural outcomes?. Current Cardiovascular Risk Reports, 11(9), 27.

Narayan, S. M., \& Zaman, J. A. (2016). Mechanistically based mapping of human cardiac fibrillation. The Journal of physiology, 594(9), 2399-2415.

Njoku, A., Kannabhiran, M., Arora, R., Reddy, P., Gopinathannair, R., Lakkireddy, D., \& Dominic, P. (2018). Left atrial volume predicts atrial fibrillation recurrence after radiofrequency ablation: a meta-analysis. Ep Europace, 20(1), 33-42.

Noh, H., Hong, S., \& Han, B. (2015). Learning deconvolution network for semantic segmentation. In Proceedings of the IEEE international conference on computer vision (pp. 1520-1528).

Papik, K., Molnar, B., Schaefer, R., Dombovari, Z., Tulassay, Z., \& Feher, J. (1998). Application of neural networks in medicine-a review. Medical Science Monitor, 4(3), MT538-MT546.

Park, C., Took, C. C., \& Seong, J. K. (2018). Machine learning in biomedical engineering.

Ren, S., He, K., Girshick, R., \& Sun, J. (2015). Faster r-cnn: Towards real-time object detection with region proposal networks. In Advances in neural information processing systems (pp. 9199).

Ronneberger, O., Fischer, P., \& Brox, T. (2015, October). U-net: Convolutional networks for biomedical image segmentation. In International Conference on Medical image computing and computer-assisted intervention (pp. 234-241). Springer, Cham.

Shahid, N., Rappon, T., \& Berta, W. (2019). Applications of artificial neural networks in health care organizational decision-making: A scoping review. PloS one, 14(2), e0212356.

Suykens, J. A., \& Vandewalle, J. (1999). Least squares support vector machine classifiers. Neural processing letters, 9(3), 293-300.

Szegedy, C., Liu, W., Jia, Y., Sermanet, P., Reed, S., Anguelov, D., ... \& Rabinovich, A. (2015). Going deeper with convolutions. In Proceedings of the IEEE conference on computer vision and pattern recognition (pp. 1-9).

Tao, Q., Ipek, E. G., Shahzad, R., Berendsen, F. F., Nazarian, S., \& van der Geest, R. J. (2016). Fully automatic segmentation of left atrium and pulmonary veins in late gadolinium-enhanced MRI: Towards objective atrial scar assessment. Journal of magnetic resonance imaging, 44(2), 346-354.

Tobon-Gomez, C., Geers, A. J., Peters, J., Weese, J., Pinto, K., Karim, R., ... \& Stender, B. (2015). Benchmark for algorithms segmenting the left atrium from 3D CT and MRI datasets. IEEE transactions on medical imaging, 34(7), 1460-1473.

Veni, Gopalkrishna, et al. "Bayesian segmentation of atrium wall using globally-optimal graph cuts on 3D meshes." International Conference on Information Processing in Medical Imaging. Springer, Berlin, Heidelberg, 2013.

Veni, G., Elhabian, S. Y., \& Whitaker, R. T. (2017). ShapeCut: Bayesian surface estimation using shape-driven graph. Medical image analysis, 40, 11-29.

Xiong, Z., Fedorov, V. V., Fu, X., Cheng, E., Macleod, R., \& Zhao, J. (2018). Fully automatic left atrium segmentation from late gadolinium enhanced magnetic resonance imaging using a 
dual fully convolutional neural network. IEEE transactions on medical imaging, 38(2), 515524.

Xiong, Z., Xia, Q., Hu, Z., Huang, N., Vesal, S., Ravikumar, N., ... \& Puybareau, E. (2020). A Global Benchmark of Algorithms for Segmenting Late Gadolinium-Enhanced Cardiac Magnetic Resonance Imaging. arXiv preprint arXiv:2004.12314.

Zhao, J., Butters, T. D., Zhang, H., Pullan, A. J., LeGrice, I. J., Sands, G. B., \& Smaill, B. H. (2012). An image-based model of atrial muscular architecture: effects of structural anisotropy on electrical activation. Circulation: Arrhythmia and Electrophysiology, 5(2), 361-370.

Zhao, J., Hansen, B. J., Wang, Y., Csepe, T. A., Sul, L. V., Tang, A., ... \& Kilic, A. (2017). Threedimensional integrated functional, structural, and computational mapping to define the structural "fingerprints" of heart-specific atrial fibrillation drivers in human heart ex vivo. Journal of the American Heart Association, 6(8), e005922. 